\title{
Aspergilosis invasiva con compromiso de tráquea
}

\author{
Invasive aspergillosis with tracheal involvement
}

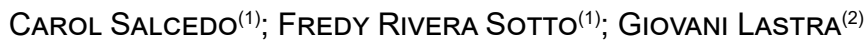

\begin{abstract}
Resumen
La aspergilosis invasiva es una causa importante de morbilidad y mortalidad en pacientes inmunocomprometidos, especialmente en aquellos que cursan con neutropenia o han sido sometidos a trasplante de médula ósea o de órgano sólido. Se expone el caso de un paciente de género masculino, de 23 años de edad, con diagnóstico previo de VIH, sin tratamiento antirretroviral, con cuadro clínico de síntomas constitucionales y respiratorios en quien se documentó aspergilosis en tráquea por hallazgos en fibrobroncospia y estudios de patología.
\end{abstract}

Palabras clave: aspergilosis, VIH.

\begin{abstract}
Invasive aspergillosis is an important cause of morbidity and mortality in immunocompromised patients, especially in those with neutropenia or those who have undergone transplantation of bone marrow or solid organs. We present the case of a 23-year-old male patient, previously diagnosed with HIV infection, without antiretroviral treatment, with a clinical picture of constitutional and respiratory symptoms, in whom aspergillosis of the trachea was documented by fibrobronchoscopy and histopathological examination.
\end{abstract}

Keywords: aspergillosis, HIV.

\section{Introducción}

Desde la aparición del VIH se ha encontrado una estrecha relación entre la enfermedad y las infecciones por microorganismos patógenos oportunistas (1). En la década de los noventa cuando se empezó a utilizar la terapia antirretroviral combinada se observó un descenso marcado en la presentación de dichas infecciones, modificándose la mortalidad de pacientes en estadio de SIDA que era del 80 al 20\% (2). De 1996 a 2012, el tratamiento antirretroviral evitó 6,6 millones de muertes relacionadas con el SIDA en todo el mundo, entre ellas 5,5 millones de muertes en países de ingresos bajos y medios (3).

A pesar de la disponibilidad del tratamiento antirretroviral, algunos pacientes no se adhieren a la terapia debido a factores psicosociales, económicos o mala tolerancia a la medicación, de ahí que persista la morbilidad de algunas patologías infecciosas como la tuberculosis, la neumonía bacteriana y la aspergilosis (4).

En la actualidad, las infecciones micóticas siguen siendo uno de los principales problemas que afrontan los pacientes con VIH; entre éstas, la
(1)Residente de Medicina Interna III año, Hospital Universitario de Neiva. (2)Docente Universidad surcolombiana. Unidad de Neumología. Hospital Universitario de Neiva.

Correspondencia: Giovani Lastra, correo electrónico: giovanilastra@yahoo.com Recibido: 16/01/14, Aprobado: 20/02/14. 
aspergillosis invasiva es la segunda micosis con mayor frecuencia de identificación, con una mortalidad del $40 \%$ y generalmente diagnosticada en $88 \%$ de los casos en los estudios postmortem (5). El objetivo de esta revisión es exponer el caso de un paciente con diagnóstico de $\mathrm{VIH}$ positivo, quien desarrolló aspergilosis invasiva con compromiso en tráquea.

\section{Caso clínico}

Paciente masculino, de 23 años de edad, con antecedente de infección por $\mathrm{VIH}$, sin tratamiento antirretroviral por falta de adherencia a este, quien ingresó al servicio de urgencias por cuadro de inicio tres meses atrás de fiebre, pérdida de peso, aparición de lesiones blanquecinas en cavidad oral, disfagia y odinofagia. Tres días previos al ingreso presentó incremento de los síntomas, acompañándose de deposiciones diarreicas y emesis. En la exploración física se observaron aftas en la cavidad oral, asociadas a lesiones violáceas y blanquecinas en faringe, adenopatías cervicales, axilares e inguinales duras y estertores pulmonares bilaterales, sin tirajes. Cursó con inestabilidad hemodinámica que requirió reanimación con líquidos endovenosos e inicio de soporte vasopresor. Posteriormente se trasladó a la unidad de cuidados intensivos, donde se inició manejo empírico con antibióticos de amplio espectro. Se tomó radiografía de tórax (figura 1), la cual mostró infiltrados intersticiales bilaterales. Los estudios de laboratorio documentaron compromiso hematológico dado por presencia de células inmaduras en sangre periférica, anisocitosis, dacriocitos, esquistocitos, poiquilocitosis y reticulocitopenia. Ante sospecha de tuberculosis pulmonar, se inició tratamiento para esta patología. Durante su evolución sufrió deterioro ventilatorio y requirió intubación orotraqueal y ventilación mecánica invasiva. Se realizó fibrobroncoscopia que evidenció lesiones blanquecinas en la tráquea y los bronquios (figura 2), de las cuales se tomaron biopsias, cuya patología reportó aspergilosis pulmonar, iniciándose tratamiento con voriconazol. Luego se obtuvieron hemocultivos positivos para Aspergillus (figura 3), además de reportes de patología que confirmaron dichos hallazgos (figuras 4 y 5).
Se practicó tomografía pulmonar en la que se evidenciaron infiltrados intersticiales bilaterales con escaso derrame pleural (figura 6).

La radiografía de tórax de control mostró empeoramiento de los infiltrados a pesar de administrarse esquema completo de tratamiento con voriconazol. El paciente evolucionó de manera tórpida y finalmente falleció.

\section{Discusión}

La aspergilosis invasiva aguda es una enfermedad rápidamente progresiva, frecuentemente fatal (6). Los pacientes que están en riesgo de aspergilosis invasiva incluyen aquellos con neutropenia prolongada, receptores de trasplantes de células madre hematopoyéticas o trasplantes de órganos sólidos y aquellos con síndrome de inmunodeficiencia adquirida avanzada o enfermedad granulomatosa crónica (6).

Generalmente la aspergilosis se presenta como un cuadro insidioso, con signos clínicos inespecíficos y en concurrencia con otras infecciones respiratorias que pueden hacer difícil el diagnóstico temprano, como ocurrió en el paciente del caso. Se han descrito tasas de mortalidad hasta del 40\% (5), que confirman que las infecciones micóticas siguen

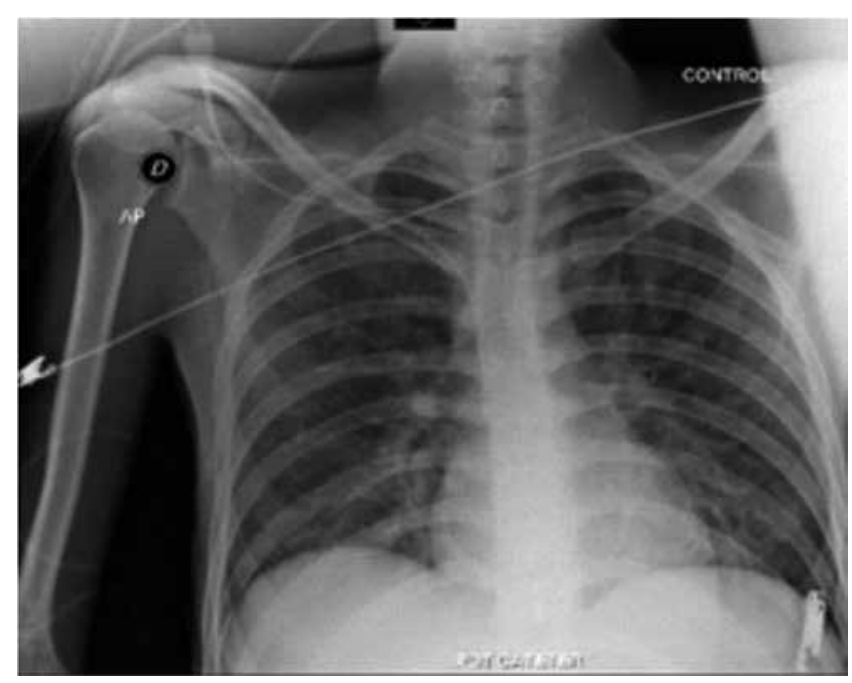

Figura 1. Radiografía de tórax en proyección PA, que evidencia infiltrados intersticiales bilaterales. 
siendo uno de los principales problemas que afrontan los pacientes inmunodeprimidos.

El Aspergillus fumigatus es la especie más común en los casos de aspergilosis invasiva aunque también se pueden encontrar Aspergillus flavus, Aspergillus niger y Aspergillus terreus (7). Puede causar afecciones que se definen clásicamente como invasivas, saprófitas o alérgicas (7). Las enfermedades oportunistas incluyen las infecciones
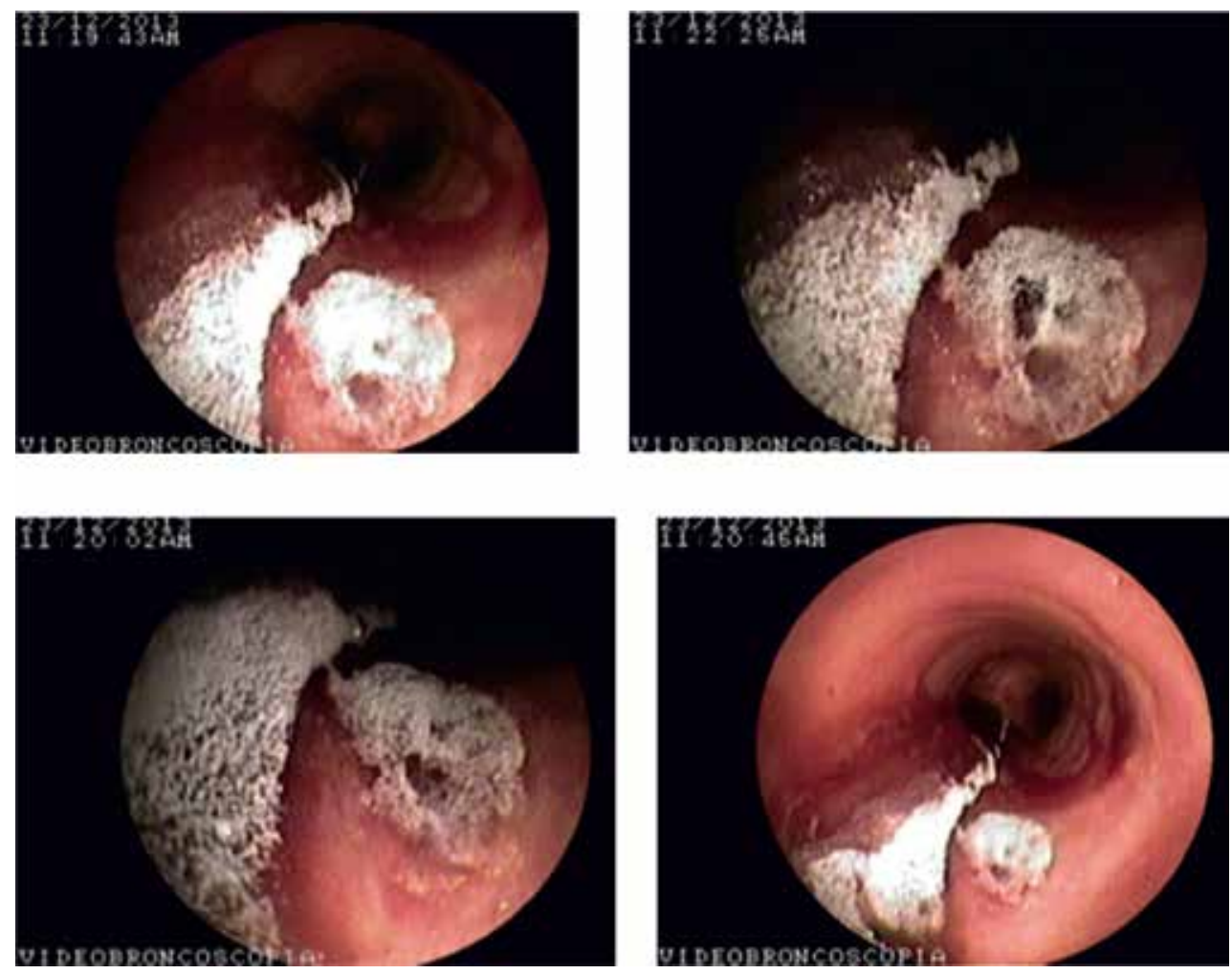

Figura 2. Imágenes de fibrobroncoscopia en la que se observan lesiones blanquecinas algodonosas que comprometen la tráquea y los bronquios.

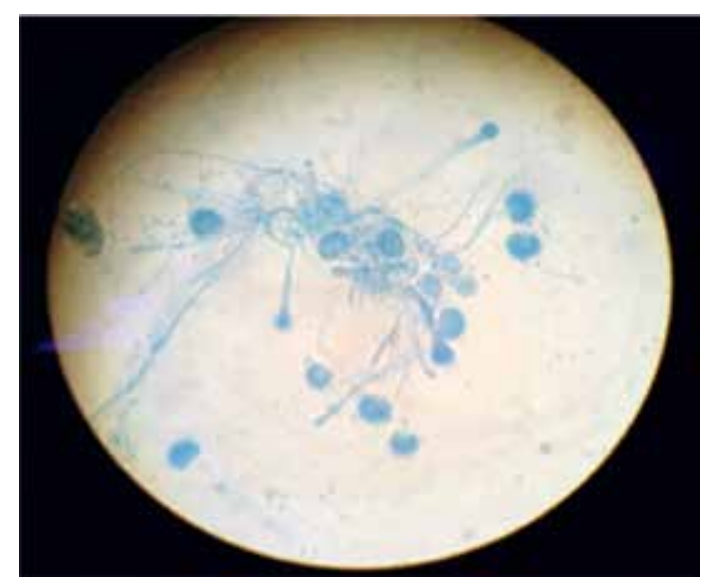

Figura 3. Hemocultivos con presencia de aspergilosis.

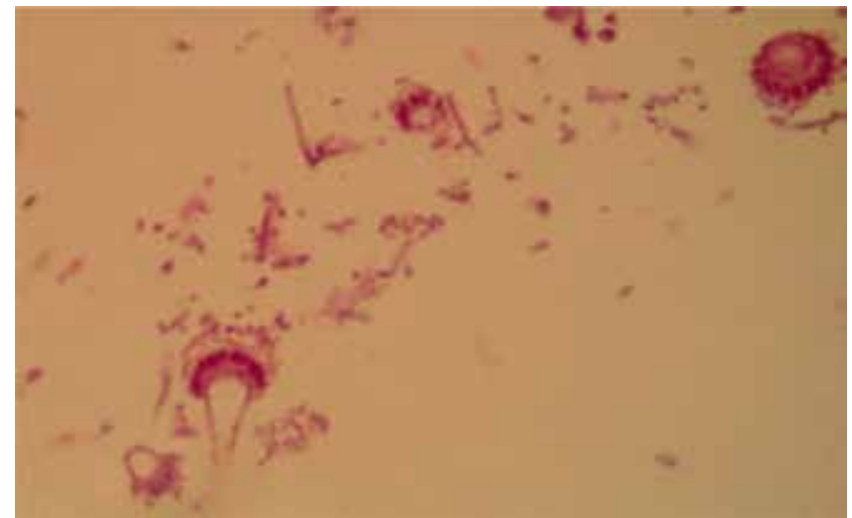

Figura 4. Biopsia traqueal con tinción de PAS. Se observan blastoconidias de Aspergillus y algunas hifas alrededor. 


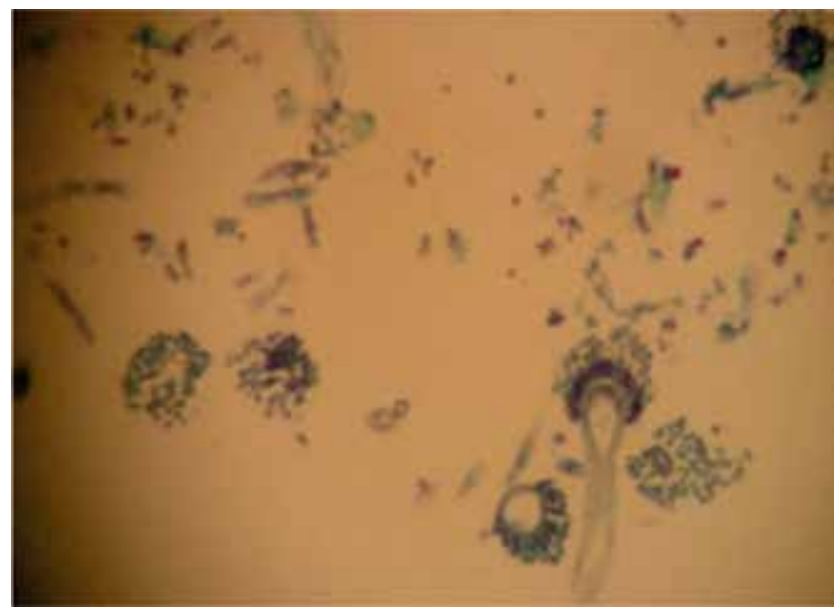

Figura 5. Biopsia traqueal. Tinción de Ziehl-Neelsen. Se observan múltiples blastoconidias de Aspergillus e hifas.

del tracto respiratorio inferior, senos paranasales y la piel como puertas de entrada (8). El sistema nervioso central, el sistema cardiovascular y otros tejidos pueden estar infectados como resultado de la diseminación hematógena o extensión directa de los focos contiguos de la infección (8). La colonización del tracto respiratorio es producto de la inhalación de las conidias del Aspergillus, y las esporas llevan a desencadenar diversos síndromes clínicos, como probablemente ocurrió en este caso.

La traqueobronquitis por Aspergillus es una forma rara pero severa de la aspergilosis pulmonar invasiva, con 150 casos publicados hasta el momento en la literatura, de los cuales, al alrededor de $75 \%$ han sido reportados en pacientes inmunocomprometidos y $45 \%$ en receptores de transplante de órgano sólido (9).

Las manifestaciones clínicas de la aspergilosis pulmonar invasiva aguda pueden ser variadas y algunos pacientes no pueden reconocer los síntomas o signos de la infección (10). Los síntomas clínicos suelen ser tos por lo general no productiva, disnea, fiebre, dolor torácico y hemoptisis (11). La progresión a neumonía con hipoxemia y disnea puede ocurrir extremadamente rápido. El dolor pleurítico y la hemoptisis sugieren infartos pulmonares y pueden ser otro tipo de patología (10). En pacien-

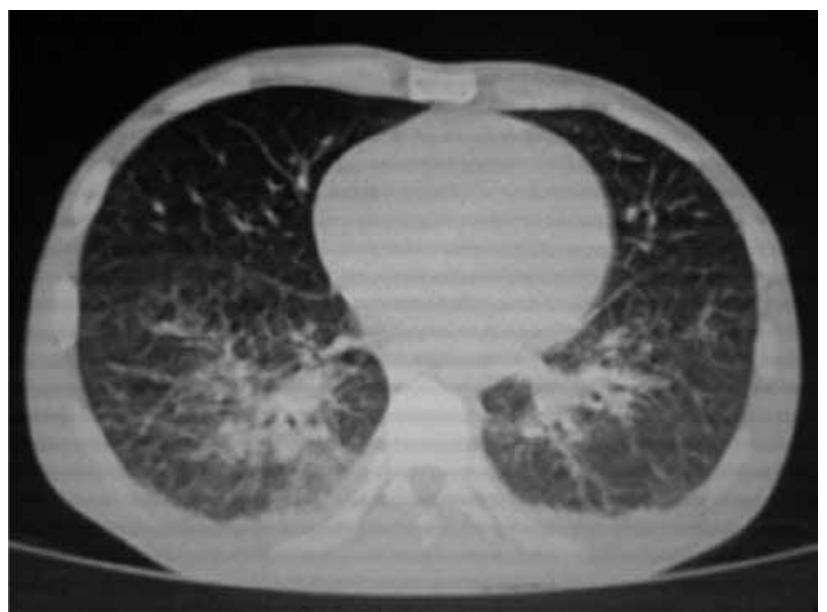

Figura 6. Tomografía de tórax de alta resolución, que muestra infiltrado mixto bilateral con derrame pleural bilateral.

tes infectados con VIH se presenta comúnmente como neumonía (11).

En la aspergilosis traqueobronquial se han descrito tres formas clínicas: la obstructiva, la ulcerosa y la pseudomembranosa (11). En el caso que se reporta, los hallazgos por fibrobroncoscopia fueron compatibles con aspergilosis pseudomembranosa. Esta forma invasiva de la enfermedad puede llevar a falla respiratoria, sepsis, perforación de la vía aérea y hemorragia fatal por fístula broncovascular (12).

Existen tres categorías de aproximación diagnóstica: infección comprobada, infección probable o infección posible, las cuales se establecen tras analizar las características de los pacientes, la patología de base, la presentación clínica, incluyendo la imagen radiológica y la documentación microbiológica o histológica (13). La evidencia histopatológica de invasión tisular por hifas y aislamiento en cultivo de Aspergillus hacen el diagnóstico de la aspergilosis invasiva comprobada. El galactomanano en suero y lavado broncoalveolar (BAL) pueden avalar el diagnóstico probable de la infección (14).

El lavado broncoalveolar, la aspiración percutánea transtorácica o la biopsia por toracoscopia son 
procedimientos estándar para establecer el diagnóstico de aspergilosis pulmonar invasiva (15). En la broncoscopia la apariencia es la de una traqueobronquitis severa que progresa a úlceras múltiples en el sitio de anastomosis. Las secuelas incluyen aspergilosis pulmonar invasiva aguda, estenosis bronquial severa y necrosis bronquial con formación de fístulas broncoarteriales. Algunos pacientes desarrollan abundantes pseudomenbranas que pueden llegar a ocluir el lumen de la vía aérea (10). En el paciente del caso se realizó fibrobroncoscopia en la que se observaron lesiones algodonosas que comprometían la tráquea y los bronquios. Las muestras de tejido y líquido obtenidas mostraron características de hifas tabicadas con dicotomía angular al examen microscópico directo y Aspergillus en los cultivos.

Con relación al tratamiento, el agente antifúngico primario para la aspergilosis invasiva es el voriconazol (16). El paciente reportado fue tratado con este medicamento pero no respondió y falleció. También se emplean las formulaciones lipídicas de anfotericina B (16). El tratamiento puede interrumpirse cuando desaparezcan los síntomas clínicos o exista resolución tanto en las imágenes radiológicas como en el estado del paciente y el recuento de células CD4 se mantenga por encima de 200 celulas/ $\mu \mathrm{L}$ (17).

\section{Conclusiones}

La infección por VIH-SIDA es un problema de salud pública que genera gran comorbilidad dado el mayor riesgo de desarrollo de infecciones oportunistas. En el caso reportado se documentó aspergilosis traqueobronquial, relacionada con la inmunosupresión. Aunque el manejo fue apropiado y siguió los lineamientos de la literatura, el pronóstico general de los cuadros de aspergilosis invasiva es ominoso en pacientes con inmunosupresión avanzada y en ausencia de terapia antirretroviral, como sucedió en el caso.

\section{Bibliografía}

1. Hoffmann, Rockstroh, Kamps. HIV Medicine 2007. 15th. Edition. Flying Publisher - Paris, Cagliari, Wuppertal.
2. Murray J. Epidemiology of human immunodeficiency virus associated pulmonary disease. Clinic Chest Med. 2013;34:165-179.

3. ONUSIDA, informe sobre la epidemia mundial de sida 2013. Disponible en: http://www.unaids.org/en/media/unaids/ contentassets/documents/ epidemiology/2013/gr2013/ UNAIDS_Global_Report_2013_es.pdf .Fecha de acceso 2/ Enero/14.

4. Guidelines for the Prevention and Treatment of Opportunistic Infections in HIV-Infected Adults and Adolescents. Disponible en: http://aidsinfo.nih.gov/guidelines. 2014. Fecha de acceso 2/Enero/14.

5. Randuz S, Kirchner C, Treckmann J, Paul A, Saner F. Aspergillus tracheobronquitis causing subtotal tracheal stenosis in a liver transplant recipient. Case Reports in Transplantation. 2013;9:282-9.

6. Segal B. Aspergillosis. N Engl J Med. 2009;360:1870-84.

7. Limper A, et al. An Official American Thoracic Society Statement: Treatment of Fungal Infections in Adult Pulmonary and Critical Care Patients. Am J Resp Crit Care Med. 2011;183:96-128.

8. Pupaibool J. Other HIV associated pneumonia. Clin Chest Med. 2013;34(2):243-54.

9. Jesús F , Yolanda M, Gema F, Santiago. Aspergilosis. Formas clínicas y tratamiento. Enferm Infecc Microbiol Clin. 2012;30:201-208.

10. Sarosi G. Fungal diseases of the lung. Aspergillus. 3rd. Edition. 1999. p. 132-133.

11. Gonzalez M, Ma Tooh M, Krueger T, Ris HB, Letovanec I, Wang Y, Lovis A. Repair of tracheal aspergillosis perforation causing tension penumothorax. Ann Thorac Surg. 2013;96:2256-8.

12. Wheat $\mathrm{J}$. Approach to the diagnosis of invasive aspergillosis and candidiasis. Clin Chest Med. 2009;30:367-77.

13. Ran Y, et al. Primary laryngeal aspergillosis related to oral sex? A case report and review of the literature. Medical Mycology Case Reports. 2013;2:1-3.

14. Lee HY, Kang HH, Kang JY, Kim SK, Lee SH, Chung YY, Kang HS, Kwon HS, Moon HS, Lee SH. A Case of tracheobronchial aspergillosis resolved spontaneously in an immunocompetent host. Tuberc Resp Dis. 2012;73:278-81.

15. Arteaga E. Aspergilosis pulmonar invasora en el síndrome de inmunodeficiencia adquirida. Rev Iberoam Micol.1999;16:211-5.

16. Tochigi N, Okubo Y, Ando T, Wakayama M, Shinozaki M, Gocho K, et al. Histopathological implications of Aspergillus infection in lung. Mediators Inflamm. 2013;2013:809798.

17. Hoffmann R. HIV medicine. 15th Edition.- Paris, Cagliari, Wuppertal: Flying Publisher; 2007. 